\title{
USO DE UNA UNIDAD DE MEDIDA INERCIAL PARA LA AYUDA AL DIAGNÓSTICO DE LA FRAGILIDAD
}

\author{
David Gualda, Juan Jesús García, Ana Jiménez, Adrián Rocandio \\ Departamento de Electrónica, Universidad de Alcalá \\ \{david.gualda, jjesus.garcia\}@uah.es \\ Dina Bousdar, Estefania Munoz Diaz \\ Instituto de Comunicaciones y Centro Alemán de Navegación Aeroespacial (DLR), Munich, Alemania \\ dina.bousdarahmed@dlr.de
}

\begin{abstract}
Resumen
En este trabajo se presenta una propuesta de contribución al diagnóstico de la fragilidad en la población anciana utilizando una Unidad de Medida Inercial (IMU) ubicada en el tobillo del paciente para obtener medidas del movimiento. El objetivo principal es utilizar esta información para medir la actividad del usuario y reconocer ciertos comportamientos como la cantidad de movimiento realizada por el paciente entre comidas, frecuencia con la que se realizan determinadas actividades diarias, etc. Posteriormente, esta información generada puede ser utilizada por el equipo médico para determinar el estado de fragilidad del paciente. Con el objetivo de verificar la viabilidad de la propuesta, se han llevado a cabo algunos experimentos iniciales con un paciente en un centro socio-sanitario.
\end{abstract}

Palabras clave: Fragilidad, IMU, reconocimiento de actividad, población anciana.

\section{INTRODUCCIÓN}

La esperanza de vida está experimentando un crecimiento constante y es por ello que en muchos países ha aumentado considerablemente la población anciana. Como consecuencia, este efecto presenta implicaciones extremas para la planificación y la prestación de asistencia sanitaria y social; especialmente cuando los ancianos dejan de ser independientes. Así, los nuevos desafíos están asociados a la mejora de la calidad de vida de estas personas, que les permitiría mantenerse independientes y llevar una vida lo más plena posible. En este sentido, la expresión utilizada para definir este deterioro causado por el envejecimiento es el estado clínico de fragilidad. La fragilidad es un síndrome geriátrico común e importante como consecuencia del declive relacionado con la edad en diversos procesos fisiológicos, que en conjunto resulta en vulnerabilidad a cambios repentinos en el estado de salud que pueden desencadenarse por alguna situación de estrés. La fragilidad, por tanto, puede conducir a una serie de resultados adversos para la salud, como discapacidad, caídas, hospitalización e incluso muerte [2].

La fragilidad se presenta en alrededor de una cuarta parte de las personas mayores de 85 años, pero diversos estudios ponen de manifiesto que es potencialmente reversible [11]. Por lo tanto, es importante identificar el estado de fragilidad para prevenir, reducir y retrasar lo máximo posible las consecuencias adversas para la salud de los adultos mayores y sus cuidadores. En consecuencia, la prevención y reducción del estado de fragilidad es uno de los retos más importantes a los que se enfrentan los organismos de salud pública de las sociedades que más envejecen [12].

El estilo de vida se considera una de las claves en el desarrollo de la fragilidad [1], y el aumento de la actividad física se ha sugerido en diversos estudios como una estrategia fundamental para prevenir la aparición y progresión de este síndrome [8]. Estudios realizados en Estados Unidos y Europa reportan que los adultos mayores pasan entre el $60 \%$ y $80 \%$ de su tiempo despiertos en comportamiento sedentario [3][10]. Por lo tanto, una comprensión más profunda de cómo el sedentarismo y la actividad física interactúan con la fragilidad en personas mayores es relevante para los profesionales de la salud a la hora de proponer estrategias para la prevención y el manejo de la fragilidad en este grupo de la población [9].

Tradicionalmente existen cinco criterios para definir el nivel de fragilidad: pérdida de peso, fatiga, lentitud al caminar, bajo nivel de actividad física y debilidad. Estos criterios son usualmente evaluados por los cuidadores a través de revisiones periódicas, siendo alguno de ellos una visión subjetiva de la persona responsable de la evaluación de la fragilidad. Sin embargo, dos de estos criterios pueden ser medidos de forma objetiva como la velocidad de la marcha y la actividad física, que son parámetros esenciales para el diagnóstico de la fragilidad.

Este trabajo forma parte de un proyecto cuyo objetivo es diseñar, desarrollar y validar un sistema que ayude a evaluar la fragilidad en pacientes de edad avanzada. Este sistema se basará en diversas tecnologías de localización que permitirán una medición suficientemente precisa de la actividad de las personas 
mayores y, también, a través de sistemas de localización, se podrán obtener sus rutinas.

Desde el punto de vista tecnológico, se propone que los pacientes porten un sistema inercial mínimamente invasivo, e incluso que puedan llevar una red de sensores corporales que permita la medición de parámetros fisiológicos relevantes para el diagnóstico. Estos sistemas se fusionarán con los sistemas de posicionamiento locales ubicados en la infraestructura (sistemas propios, redes $\mathrm{WiFi}$, etc.) del entorno (centro geriátrico, hospital e incluso domicilio del paciente).

El objetivo es proporcionar al personal médico y sanitario un conjunto de evidencias objetivas que faciliten la evaluación precoz de la fragilidad de los pacientes, durante los periodos de tiempo que estén en tratamiento médico, así como el seguimiento de terapias establecidas que permitan retrasar e incluso revertir la aparición de fragilidad.

Como primera etapa de esta investigación, proponemos que la persona lleve una Unidad de Medida Inercial (IMU), no sólo para obtener los datos con el objetivo de calcular la velocidad de marcha y el gasto energético, sino también para obtener información sobre las actividades diarias del usuario. La recogida de datos durante un tiempo prolongado proporcionará un patrón de comportamiento y una estimación de la relación actividad-tiempo sedentaria, que será muy útil para que el personal de asistencia evalúe el grado de fragilidad y su evolución en el paciente.

El resto de partes de este artículo están organizadas de la siguiente forma: La Sección 2 define los métodos y materiales utilizados en este trabajo; en la Sección 3 se detallan los resultados preliminares obtenidos; y por último, en la Sección 4 se muestran las conclusiones.

\section{MÉTODOS Y MATERIALES}

En esta sección se presenta el dispositivo de medida inercial utilizado para registrar la información relacionada con el paciente, su ubicación corporal y el procesamiento de las salidas de la IMU con el fin de obtener información de las actividades del paciente.

\subsection{SELECCIÓN DE LA UBICACIÓN DE LA IMU EN EL CUERPO}

El nivel de detalle respecto a la monitorización de la actividad de un paciente depende de la ubicación corporal del sensor inercial. La posición de éste determinará los aspectos de la actividad física que se pueden medir. Por ejemplo, algunas posiciones del cuerpo permiten detectar sólo pasos por lo que entonces únicamente se pueden determinar los tiempos de actividad e inactividad. Otras ubicaciones corporales del sensor podrían permitir distinguir entre caminar, subir y bajar por las escaleras y cuando el usuario se encuentra parado o sentado. Dicha información proporciona un valor añadido para evaluar la actividad física del paciente. Para los experimentos llevados a cabo en este trabajo se han testeado dos ubicaciones del cuerpo: la parte exterior del bolsillo del pantalón (en la parte superior de la pierna) y el tobillo. La figura 1 muestra la posición de la IMU en estas dos ubicaciones.

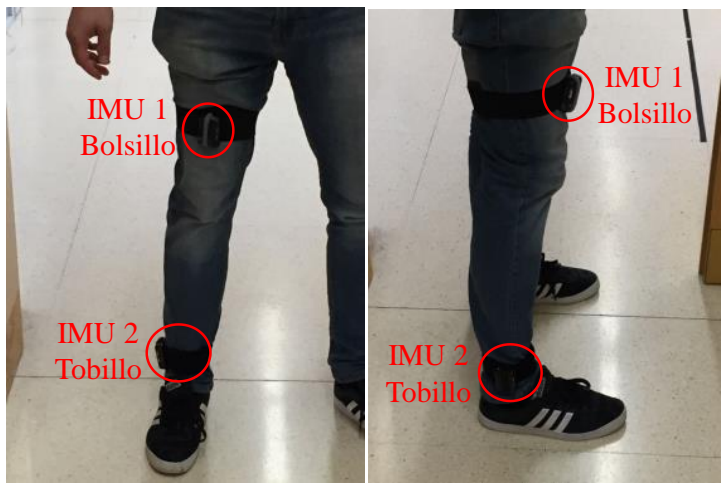

Figura 1: Localización corporal de las IMUs, en el bolsillo y en el tobillo.

\subsection{MATERIALES}

Para este trabajo de investigación, se ha seleccionado la unidad de medida inercial GIMU, de X-IO [14] (ver figura 2). Este dispositivo proporciona información de acelerómetro y giróscopo de 3 ejes así como la presión a través de un barómetro integrado en el dispositivo, a una frecuencia de muestreo configurable (en este caso se ha ajustado a $100 \mathrm{~Hz}$ ). Además, esta IMU es capaz de almacenar información en una tarjeta de memoria SD y gracias a su batería de $1000 \mathrm{mAh}$ permite almacenar de forma continuada 12 horas de datos aproximadamente, lo cual es suficiente para monitorizar al paciente durante el día.

\subsection{MÉTODOS}

En este trabajo, la actividad del paciente (distancia recorrida, número de pasos, gasto energético, etc.) es obtenida a partir de los ángulos de Euler de la IMU. Estos ángulos son balanceo, cabeceo y guiñada, que se corresponden con Roll, Pich y Yaw en inglés. En este caso, las orientaciones de estos ángulos de la IMU se muestran en la figura 2.

Por otra parte, la figura 3 muestra la estimación de los ángulos de Euler a partir de los datos del acelerómetro y giróscopo, utilizando para ello un Filtro de Kalman Extendido (EKF), como se detalla en [6].

La monitorización de actividad basada en la detección de pasos se realiza identificando picos del ángulo de Euler correspondiente, siendo el ángulo Pitch cuando la IMU se encuentra en la parte exterior del bolsillo y el Roll en el caso del tobillo, ya que estos ángulos siguen el movimiento de la pierna cuando el paciente 
está caminando (para el caso del tobillo, si la IMU está situada en la parte delantera de la pierna debemos considerar el ángulo Pitch).

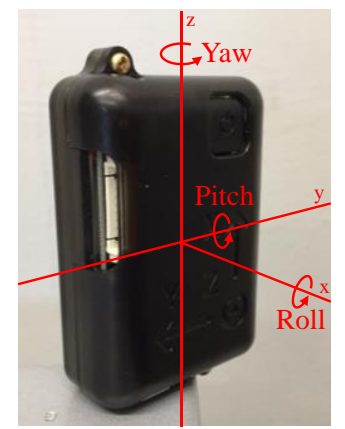

Figura 2: IMU utilizada y representación de los ángulos de Euler.

Esta detección de actividad se basa en el trabajo desarrollado en [4], en el que la ubicación de la IMU fue el bolsillo. En dicho trabajo, el detector analiza la señal Pitch para identificar los pasos.

Entradas

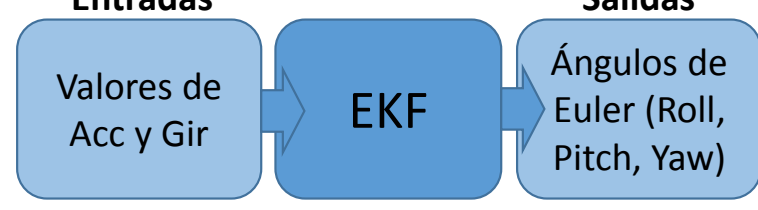

Figura 3: Proceso de estimación de los ángulos de Euler utilizando un EKF.

Para evitar falsos positivos, únicamente se detecta un paso si la amplitud de la señal Pitch está por encima de un umbral previamente definido.

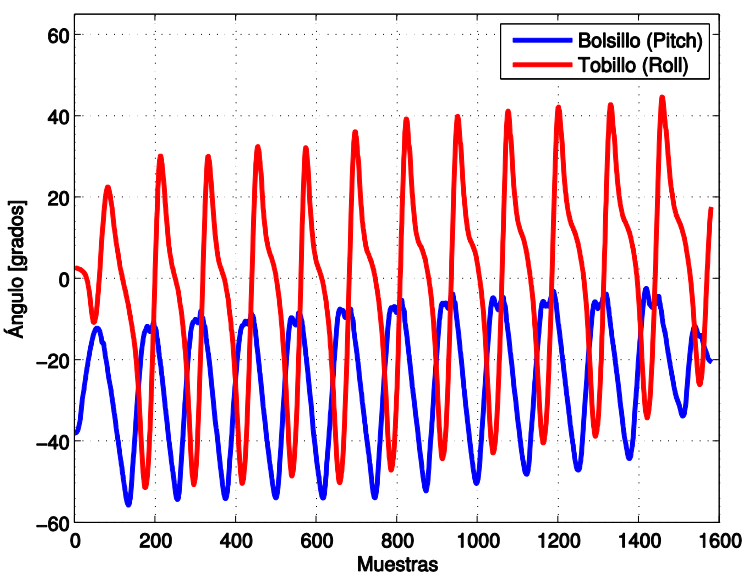

Figura 4: Ejemplo de las señales utilizadas para la detección de pasos (Pitch para la IMU localizada en el bolsillo y Roll para la IMU del tobillo).

Aunque este algoritmo de detección de pasos fue diseñado originalmente para la IMU ubicada en el bolsillo, puede ser adaptado para detectar pasos con la IMU localizada en el tobillo o en el pie.

La figura 4 muestra un ejemplo de la estimación de estas señales (Pitch para IMU1 y Roll para la IMU2, localizadas en el bolsillo $y$ en el tobillo, respectivamente) para una distancia recorrida de unos $17 \mathrm{~m}$. Nótese que ambas señales son independientes por lo que no están sincronizadas. No obstante, la detección de paso ocurre aproximadamente en el mismo instante de tiempo.

Como se puede apreciar, en ambos casos las señales son lo suficientemente claras para detectar cada paso del paciente. No obstante, la señal procedente de la IMU situada en el tobillo es la de mayor amplitud.

Con respecto a la estimación de la distancia de paso, se ha seguido la propuesta desarrollada en [4], en la que dicha distancia se obtiene como un valor dependiente de la amplitud de paso, de forma que se establece una relación lineal entre la distancia y esta amplitud, como se muestra en la expresión (1):

$$
\hat{d}=b \cdot A+c
$$

La variable $A$ representa la amplitud de la señal relacionada con la detección de un solo paso; $b$ es un parámetro común que se obtiene mediante un proceso de calibración; y $c$ es un parámetro particularizado para cada paciente. Siguiendo este método se han calibrado los parámetros para cada ubicación de IMU (bolsillo y tobillo) utilizando para ello 10 usuarios, recorriendo una distancia conocida de $17.6 \mathrm{~m}$.

A partir de esta prueba, se ha obtenido un parámetro $b$ común y un valor $c$ para cada persona.

Para resolver el problema de optimización se ha utilizado un algoritmo meta-heurístico denominado Harmony Search (HS) [5], cuyo rendimiento para resolver este tipo de problemas se ha demostrado en un gran número de estudios y aplicaciones [13]. Sin embargo, se pueden utilizar otros métodos de optimización, como por ejemplo los que se basan en Algoritmos Genéticos (GA) [7].

En las tablas 1 y 2 se muestran los resultados de la calibración de las IMUs para 10 usuarios.

Tabla 1: Parámetros de la IMU ubicada en el bolsillo.

\begin{tabular}{|c|c|c|c|}
\hline Usuario & $b$ & $C$ & $\begin{array}{l}\text { Error medio } \\
\text { (m) }\end{array}$ \\
\hline 1 & \multirow{10}{*}{0.0192} & 0.6412 & \multirow{10}{*}{0.43} \\
\hline 2 & & 0.3689 & \\
\hline 3 & & 0.5500 & \\
\hline 4 & & 0.8241 & \\
\hline 5 & & 0.5109 & \\
\hline 6 & & 0.6326 & \\
\hline 7 & & 0.4478 & \\
\hline 8 & & 0.6941 & \\
\hline 9 & & 0.7390 & \\
\hline 10 & & 0.6932 & \\
\hline
\end{tabular}


Tabla 2: Parámetros de la IMU ubicada en el tobillo.

\begin{tabular}{|c|c|c|c|}
\hline Usuario & $b$ & $c$ & $\begin{array}{l}\text { Error medio } \\
(\mathrm{m})\end{array}$ \\
\hline 1 & \multirow{10}{*}{0.0149} & 0.2975 & \multirow{10}{*}{0.41} \\
\hline 2 & & 0.0387 & \\
\hline 3 & & 0.5418 & \\
\hline 4 & & 0.4227 & \\
\hline 5 & & 0.3232 & \\
\hline 6 & & 0.1720 & \\
\hline 7 & & 0.1556 & \\
\hline 8 & & 0.2862 & \\
\hline 9 & & 0.2693 & \\
\hline 10 & & 0.3320 & \\
\hline
\end{tabular}

\section{RESULTADOS EXPERIMENTALES}

Se ha realizado una prueba experimental en un centro geriátrico con un paciente real. En este caso, el usuario es una mujer de 88 años que utiliza un andador para desplazarse por el edificio. Para su monitorización hemos utilizado una IMU colocada en el tobillo (ver figura 5), ya que es menos invasiva y más cómoda que la ubicación en la parte superior de la pierna, sobre todo si se trata de una anciana.

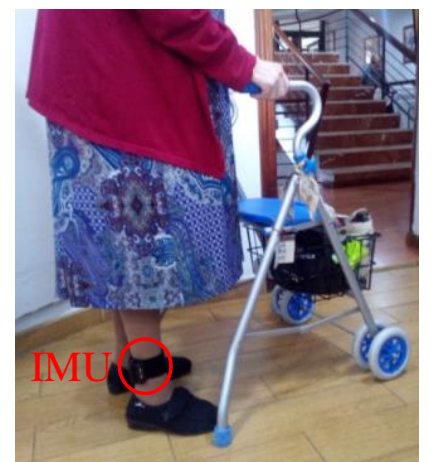

Figura 5: Paciente mayor con la IMU ubicada en el tobillo.

Este primer experimento ha consistido en monitorizar a la paciente durante el día. En la tabla 3 se detalla su rutina habitual y la ubicación por planta de cada una de las actividades que realiza (nótese que su habitación se encuentra en la planta 2).

Los resultados para un solo día (casi 13 horas) se muestran en la figura 6.

Se puede observar que el resultado de la medición del "Roll" presenta cambios bruscos en la señal debido a movimientos normales repentinos del tobillo de la paciente, lo cual, hace difícil la detección de pasos. Es por ello que se ha implementado un algoritmo de postprocesado para filtrar la señal y eliminar los saltos. Dicho algoritmo consiste en calcular la diferencia entre la señal y una media móvil, resultando en la señal filtrada que denominamos como "Roll Procesado".
Tabla 3: Secuencia habitual de actividades de la paciente durante el día.

\begin{tabular}{|c|c|c|}
\hline Secuencia & Actividad & Planta \\
\hline 1 & Desayuno & -1 \\
\hline 2 & Gimnasio & 1 \\
\hline 3 & Habitación & 2 \\
\hline 4 & Comida & -1 \\
\hline 5 & Habitación & 2 \\
\hline 6 & Cena & -1 \\
\hline 7 & Habitación & 2 \\
\hline
\end{tabular}
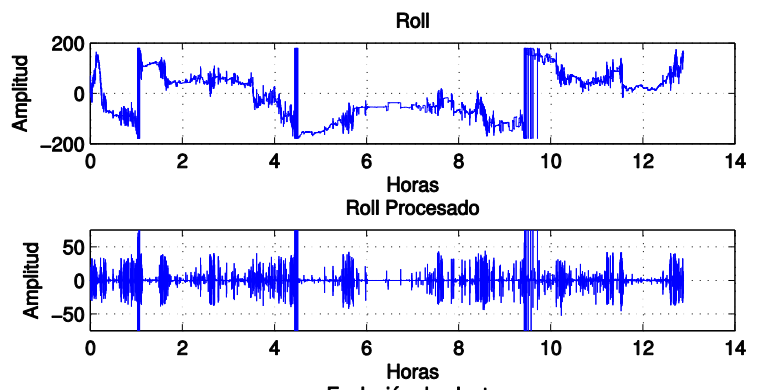

Evolución de planta

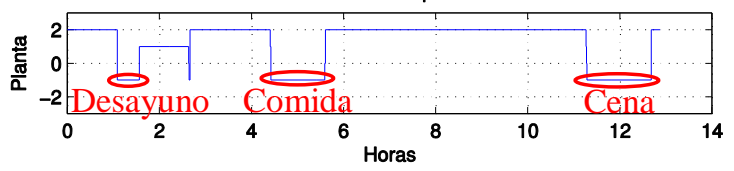

Figura 6: Resultados de monitorización de la paciente para un día.

A partir de la señal de "Roll Procesado", el número de pasos detectados fue de 1510 , por lo que la distancia recorrida aproximada a lo largo del día del experimento según la expresión (1) fue de 1360m (se ha utilizado el parámetro $c=0.2635$ asociado a la paciente y el parámetro general $b$ que se muestra en la tabla 2). Además, se puede observar claramente en la información proporcionada por el barómetro (imagen inferior de la figura 6) que en los intervalos en los que la paciente se movía entre plantas (siempre haciendo uso del ascensor) su actividad cambia. Por lo tanto, estos resultados se pueden considerar como un punto de partida adecuado de la investigación que se propone, ya que es posible medir la actividad del paciente durante el día, así como identificar de manera aproximada la actividad que el usuario está realizando entre intervalos gracias a la detección de cambios de planta.

El siguiente paso de esta investigación es obtener la información inercial del paciente durante varias semanas consecutivas para generar información estadística relacionada con la cantidad de movimiento y el gasto de energía, identificando algunos intervalos de actividad, para ayudar a detectar cambios en los parámetros relacionados con las actividades del paciente $\mathrm{y}$, en consecuencia, tener información objetiva para identificar el estado de fragilidad del usuario por parte de los médicos. 


\section{CONCLUSIONES}

En este trabajo se ha presentado una propuesta para proporcionar información objetiva al personal médico con el fin de ayudar a diagnosticar el estado de fragilidad en la población anciana. Esta información está relacionada con el movimiento y la actividad del paciente durante el día, como el número de pasos, la distancia recorrida, la velocidad media, los cambios de ubicación, etc. Los resultados experimentales demuestran que es posible generar dicha información, monitorizando al paciente durante el día, con el objetivo futuro de obtener un alto número de datos en días consecutivos para generar información estadística que sirva de ayuda en la detección de signos que sugieran un estado de fragilidad. El objetivo final es generar patrones de comportamiento de los pacientes que puedan ser útiles para mejorar el diagnóstico realizado por los médicos.

\section{Agradecimientos}

El desarrollo de este trabajo ha sido posible gracias a la Universidad de Alcalá (proyectos Echo-Drone3D, CCGP2017-EXP/50, LocActiv, CCGP2017EXP/053, y UAH-AE2017-4), y al Ministerio de Economía y Competitividad (TARSIUS, TIN201571564-C4-1-R). Además, los autores de este trabajo agradecen la colaboración de los cuidadores de la residencia Albertia-Las Palmeras, especialmente a las residentes voluntarias $\mathrm{M}$. y L. por su ayuda en la realización de este estudio.

\section{English summary}

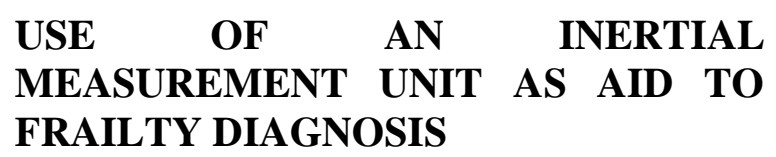

\begin{abstract}
This paper presents a proposal for contributing to the diagnosis of frailty in the elderly people using an Inertial Measurement Unit (IMU) located at the ankle of the patient in order to obtain measurements of movement. The main objective is to use this information to measure the activity of the user and to recognize certain behaviors such as the amount of movement carried out by the patient between meals, frequency in which certain daily activities are performed, etc. This generated information can be used by the medical staff to determine the patient's frailty state. In order to verify the feasibility of the proposal, some initial experiments have been carried out with a real patient in a geriatric center.
\end{abstract}

Keywords: Frailty, IMU, Elderly people, activity recognition.

\section{Referencias}

[1] Bergman, H., Ferrucci, L., Guralnik, J., Hogan, D. B., Hummel, S., Karunananthan, S., Wolfson, C., (2007) "Frailty: An Emerging Research and Clinical Paradigm--Issues and Controversies", The Journals of Gerontology Series A: Biological Sciences and Medical Sciences, vol. 62, no. 7, pp. 731-737.

[2] Clegg, A., Young, J., Iliffe, S., Rikkert, M. O., Rockwood, K., (2013) "Frailty in elderly people", The Lancet, vol. 381, no. 9868, pp. 752762.

[3] Davis, M. G., Fox, K. R., Hillsdon, M., Sharp, D. J., Coulson, J. C., Thompson, J. L., (2011) "Objectively Measured Physical Activity in a Diverse Sample of Older Urban UK Adults" Medicine \& Science in Sports \& Exercise, vol. 43, no. 4, pp. 647-654.

[4] Diaz, E. M., Gonzalez, A. L. M., (2014) "Step detector and step length estimator for an inertial pocket navigation system", International Conference on Indoor Positioning and Indoor Navigation (IPIN).

[5] Geem, Z. W., Kim, J. H., Loganathan, G. V., (2001) "A New Heuristic Optimization Algorithm: Harmony Search", Simulation, vol. 76 , no. 2 , pp. 60-68.

[6] Gualda, D., Díaz, E., García, J. J., Pérez, M. C., Ureña, J., Cervigón, R., (2017) "Patient Activity Monitoring for Smartphones based on a PDR Algorithm", International Conference on Indoor Positioning and Indoor Navigation (IPIN).

[7] Holland, J. H., (1975) "Adaption in Natural and Artificial Systems", Ann Arbor: The University of Michigan Press.

[8] Liu, C. K., Fielding, R. A., (2011) "Exercise as an Intervention for Frailty", Clinics in Geriatric Medicine, vol. 27, no. 1, pp. 101-110.

[9] Mañas, A., del Pozo-Cruz, B., Guadalupe-Grau, A., Marín-Puyalto, J., Alfaro-Acha, A., Rodríguez-Mañas, L., García-García, F. J., Ara, I., (2018) "Reallocating AccelerometerAssessed Sedentary Time to Light or Moderateto Vigorous-Intensity Physical Activity Reduces Frailty Levels in Older Adults: An Isotemporal 
Substitution Approach in the TSHA Study", Journal of the American Medical Directors Association, vol. 19, no. 2, pp. 185.e1-185.e6.

[10] Matthews C. E., Chen, K. Y., Freedson, P. S., Buchowski, M. S., Beech, B. M., Pate, R. R., Troiano, R. P., (2008) "Amount of Time Spent in Sedentary Behaviors in the United States, 20032004", American Journal of Epidemiology, vol. 167, no. 7, pp. 875-881.

[11] Ng, T. P., Feng, L., Nyunt, M. S. Z., Feng, L., Niti, M., Tan, B. Y., Chan, G., Khoo, S. A., Chan, S. M., Yap, K. B., (2015) "Nutritional, Physical, Cognitive, and Combination Interventions and Frailty Reversal Among Older Adults: A Randomized Controlled Trial", The American Journal of Medicine, vol. 128, no. 11, pp. 1225-1236.e1.

[12] Puts, M. T. E., Toubasi, S., Atkinson, E., Ayala, A. P., Andrew, M., Ashe, M. C., Bergman, H.,
Ploeg, J., McGilton, K. S., (2016) "Interventions to prevent or reduce the level of frailty in community-dwelling older adults: a protocol for a scoping review of the literature and international policies", BMJ Open, vol. 6, no. 3, p. e010959.

[13] Wang, X., Gao, X.-Z., Zenger, K., (2014) “The Overview of Harmony Search", SpringerBriefs in Applied Sciences and Technology, pp. 5-11.

[14] X-IO, NGIMU (2018) Available online: http://xio.co.uk/ngimu/ (accessed on 28 June 2018).

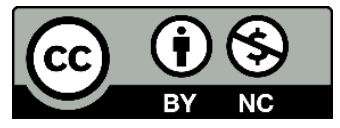

(C) 2018 by the authors. Submitted for possible open access publication under the terms and conditions of the Creative Commons Attribution $\quad \mathrm{CC}-\mathrm{BY}-\mathrm{NC} \quad 3.0 \quad$ license (https://creativecommons.org/licenses/by-nc/3.0). 